\title{
ACTIVIDADES ANTIHIPERTENSIVA Y TÓXICA DEL EXTRACTO METANÓLICO DE Calceolaria myriophylla “ZAPATILLA” EN RATONES CON HIPERTENSIÓN INDUCIDA POR L-NAME
}

\author{
Antihypertensive and tóxica activities of methanolic extract of Calceolaria myriophylla \\ "zapatilla" in mice with hypertension induced by L-NAME \\ Martin Condorhuamán ${ }^{1}$, Jorge L. Arroyo ${ }^{2}$, Ruth R. Cuba ${ }^{3}$ \\ ${ }^{1}$ Instituto de Investigación en Ciencias Farmacéuticas y Recursos Naturales "Juan de Dios Guevara", Facultad de Farmacia y Bioquímica. \\ ${ }^{2}$ Facultad de Medicina. ${ }^{3}$ Facultad de Farmacia y Bioquímica. Universidad Nacional Mayor de San Marcos
}

\section{RESUMEN}

El presente estudio experimental tuvo como objetivo determinar las actividades antihipertensiva y tóxica del extracto metanólico de Calceolaria myriophylla (zapatilla). Se utilizaron ratones albinos machos Mus musculus BALB/c, de dos meses con peso de $25 \pm 5$ gramos para la inducción de hipertensión arterial con la administración de N-nitro-L-arginina metíl eter (L-NAME) a dosis de 13o $\mathrm{mg} / \mathrm{kg} /$ día, vía oral durante siete días, midiéndose la presión arterial basal, y post inducción. Los ratones fueron divididos en seis grupos de diez animales: grupo control negativo (agua destilada), control positivo (L-NAME), grupos experimentales a dosis de 200 , 400 y $600 \mathrm{mg} / \mathrm{kg}$ del extracto metanólico, y enalapril a $75 \mathrm{mg} / \mathrm{kg}$. Se administró el tratamiento durante 30 días continuos, midiendo la presión arterial cada cinco días. El estudio de toxicidad crónica se determinó a dosis repetidas por 6o días en ratas albinas machos de raza Holtzmann con peso de $180 \pm 20$ gramos, normotensas, distribuidas en tres grupos de seis animales cada uno, utilizando dosis de 400 y $600 \mathrm{mg} / \mathrm{kg}$. El extracto metanólico de Calceolaria myriophylla presentó abundante cantidad de compuestos fenólicos, mejor efecto antihipertensivo a dosis de $600 \mathrm{mg} / \mathrm{kg}$ comparado con enalapril y no presentó toxicidad crónica a las dosis evaluadas; concluyéndose que presentó actividad antihipertensiva sin efectos tóxicos.

Palabras clave: Calceolaria myriophylla, actividad antihipertensiva, toxicidad crónica, ratones albinos.

\section{SUMMARY}

Objective of this experimental study was to determine the antihypertensive and toxic activities of methanolic extract from Calceolaria myriophylla (zapatilla). Male albino mice Mus musculus BALB/c of two months, $25 \pm 5$ g weigth, were used for induction of hypertension with N-nitro-L-arginine methyl ester (L-NAME) administered at doses of $130 \mathrm{mg} / \mathrm{kg} /$ day, orally for seven days, measuring baseline and post induction blood pressure. The mice were divided into six groups of ten animals: negative control (distilled water), positive control (L-NAME), experimental groups at doses of 200,400 and $600 \mathrm{mg} / \mathrm{kg}$ of methanolic extract, and enalapril group to doses $75 \mathrm{mg} / \mathrm{kg}$. The treatment was administered for 30 days continuous and blood pressure was measured every five days. The study of chronic toxicity was determined to repeated doses for 60 days in normotensive rats, divided into three groups of six animals each group; using doses of 400 and $600 \mathrm{mg} / \mathrm{kg}$. The methanolic extract from Calceolaria myriophylla presented abundance of phenolics compounds, better antihypertensive effect at the dose of $600 \mathrm{mg} / \mathrm{kg}$ compared with enalapril and it did not show chronic toxicity at the doses evaluated; concluding that the methanolic extract showed antihypertensive activity without toxic effects.

Keywords: Calceolaria myriophylla, antihypertensive activity, chronic toxicity, albino mice.

\section{INTRODUCCIÓN}

a hipertensión arterial (HTA) es uno de los principales factores de riesgo cardiovascular y causa directa de morbilidad y mortalidad en los países desarrollados, estando implicada en la enfermedad isquémica cardíaca, insuficiencia cardíaca, enfermedad vascular cerebral, nefropatías y alteraciones orgánicas y funcionales originadas por lesiones microvasculares 1; por lo tanto, el tratamiento antihipertensivo previene complicaciones cardiovasculares, cerebrovasculares y renales, pudiendo incluir el control de factores de riesgo y de enfermedades asociadas que generan mayor riesgo. En la actualidad existe diversidad de esquemas de tratamiento, pero la mayor parte presentan efectos secundarios y sus costos determinan que el paciente, por lo general, no continúe con su terapia ${ }^{2}$.

Calceolaria myriophylla es una hierba anual de 20 a $30 \mathrm{~cm}$ de altura, cuyas flores amarillas son parecidas a las "zapatillas o zapatos”, de ahí su nombre común. Se encuentra en laderas y cercos de piedra hasta los 4000 metros de altitud ${ }^{3}$. Las flores tienen corola bilabiada con la parte inferior hinchada, la inflorescencia tiene 3-10 flores, las ramas son blancas y las hojas son pequeñas, elípticas a ovaladas ${ }^{4}$ (figura 1). Existen múltiples reportes etnobotánicos de sus propiedades terapéuticas, entre las que destacan el tratamiento de afecciones renales y vías urinarias, reumatismos, regulaciones menstruales, infecciones, úlceras gástricas y dolores estomacales; sin embargo, existen escasos estudios científicos que validen estas aplicaciones ${ }^{3-5}$. La presente investigación farmacológica tuvo como objetivo evaluar el actividad antihipertensiva y tóxica del extracto etanólico de esta especie en ratones con hipertensión inducida por L-NAME, para sustentar su posible uso como terapia alternativa o coadyuvante al tratamiento de la hipertensión arterial. 


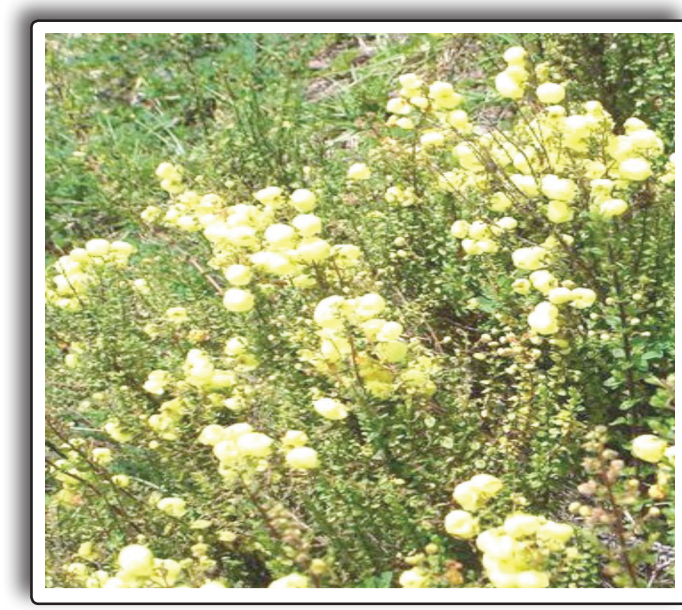

Figura 1. Calceolaria myriophylla "Zapatilla".

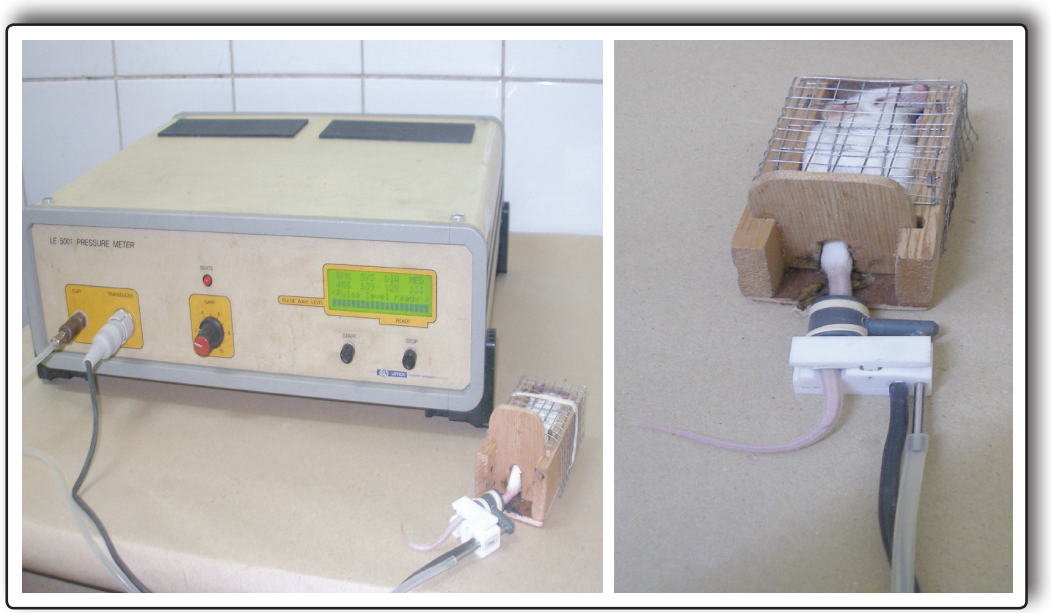

Figura 2. Medición incruenta de la presión arterial

\section{MATERIALES Y MÉTODOS}

Es una investigación experimental de tipo descriptivo, analítico, prospectivo y longitudinal, realizada en la Facultad de Medicina de la Universidad Nacional Mayor de San Marcos.

\section{Material biológico}

Se utilizaron ratones albinos machos Mus musculus BALB/c de 2 meses, con peso de $25 \pm 5 \mathrm{~g}$, y ratas albinas macho de raza Holtzmann con peso de $180 \pm 20 \mathrm{~g}$.

\section{Recolección y obtención de la muestra vegetal}

La planta en estudio fue recolectada en el distrito de Q'orao (350o metros de altitud), provincia del Cusco, región Cusco. Se colectó la planta entera en su estado silvestre (hojas, tallos y flores) sin raíz. La muestra se sometió a secado en un horno a temperatura constante de $35^{\circ} \mathrm{C}$, luego se realizó la molienda y finalmente el tamizaje para uniformizar el pulverizado.

\section{Obtención del extracto metanólico y análisis fitoquímico}

El extracto se obtuvo en una proporción de $20 \%(\mathrm{P} / \mathrm{V})$ en metanol, para lo cual se procedió a la maceración de la muestra seca pulverizada por 15 días, pasando el macerado a filtración y luego a evaporación para obtener el extracto seco ${ }^{6}$, que fue conservado de 4 a $8^{\circ} \mathrm{C}$ y posteriormente sometido a análisis fitoquímico -basado en reacciones de coloración o precipitación- para la determinación de metabolitos secundarios ${ }^{7}$.

Tabla 1. Análisis fitoquímico del extracto metanólico de Calceolaria myriophylla.

\begin{tabular}{lcc}
\hline \multicolumn{1}{c}{ Reactivos } & $\begin{array}{c}\text { Metabolitos } \\
\text { Secundarios }\end{array}$ & Reacción \\
\hline Reacción con tricloruro férrico 1\% & Compuestosfenólicos & +++ \\
Reacción de Shinoda & Flavonoides & ++ \\
Reacción de la gelatina - sal 1\% & Taninos & + \\
Reacción de Dragendorff & Alcaloides & + \\
Reacción de Mayer & Alcaloides & + \\
Reacción de hidróxido de sodio & Quinonas & + \\
Reacción de Molish & Glicósidos & - \\
\hline
\end{tabular}

(+++) Abundante cantidad, (++) Regular cantidad,

$(+)$ Poca cantidad, (-) Ausente.
Determinación de la actividad antihipertensiva en ratones

Para la medición de la presión arterial se utilizó un medidor de presión arterial incruento, que es un instrumento con memoria basado en un microprocesador específico diseñado para realizar la medición indirecta de la presión sanguínea en animales de experimentación (figura 2). La inducción de la hipertensión arterial se hizo administrando N-nitro-L-arginina metíl ester (L-NAME) a dosis de $130 \mathrm{mg} / \mathrm{kg} /$ día, vía oral, durante siete días, en ratones albinos, con monitoreo de la presión arterial sistólica, diastólica y media, en los estadíos basal y post inducción ${ }^{8-9}$.

Los animales fueron mantenidos en jaulas de crianza acondicionados al ambiente de laboratorio con un ciclo de luz-oscuridad de 12 horas a temperatura de $24 \pm 2^{\circ} \mathrm{C}$. 6o ratones, pesados, distribuidos al azar en seis grupos de diez animales cada uno, y marcados, fueron privados de agua y alimentos 12 horas antes del inicio de procedimientos, al cabo de las cuales se les midió la presión arterial basal antes de la inducción con L-NAME. Los grupos formados fueron: 1: agua destilada; 2: L-NAME (130 mg/kg) ${ }^{10}$; 3, 4 y 5: L-NAME + extracto metanólico a dosis de 200, 400 y $600 \mathrm{mg} / \mathrm{kg}$, respectivamente; y 6 : $\mathrm{L}-\mathrm{NAME}$ + enalapril a dosis de $75 \mathrm{mg} / \mathrm{kg}$.

La presión arterial se midió colocando el sensor en el tercio medio de la cola del animal con previa dilatación a una temperatura de 30 a $35^{\circ} \mathrm{C}$. En cada sesión se registró el promedio de tres mediciones por ratón. Se hicieron en total 6 sesiones con cada espécimen -una cada cinco días, durante un periodo de 30 días-). Los datos de presión arterial sistólica, diastólica y media fueron expresados en $\mathrm{mmHg}$.

\section{Determinación de la toxicidad crónica}

Los animales de experimentación, adaptados a las condiciones de laboratorio, fueron marcados y pesados periódicamentehastalaculminacióndelensayo. Seutilizaron 24 ratas divididas al azar en tres grupos de ocho animales: 1: control agua destilada; 2 y 3: extracto metanólico a dosis de 400 y $600 \mathrm{mg} / \mathrm{kg}$, respectivamente. La administración, en 
Tabla 2. Efecto antihipertensivo sobre la presión arterial sistólica del extracto metanólico en ratones con hipertensión inducida por L-NAME.

\begin{tabular}{|c|c|c|c|c|c|c|c|}
\hline Tratamiento & PB & PPI & PS1 & PS5 & PS10 & PS15 & PS20 \\
\hline Control sin L-NAME & $106,24 \pm 1,23$ & $105,34 \pm 0,66^{\mathrm{a}}$ & $104,7 \pm 1,76$ & $106,17 \pm 1,21$ & $108,86 \pm 1,83$ & $110,83 \pm 1,47$ & $110,93 \pm 1,10$ \\
\hline Control con L-NAME & $107,07 \pm 1,09$ & $161 \pm 1,33$ & $161,7 \pm 1,40^{\mathrm{b}}$ & $154,9 \pm 0,72^{b}$ & $147,56 \pm 1,33^{b}$ & $139,84 \pm 1,51^{b}$ & $126,66 \pm 1,19^{b}$ \\
\hline Extracto 200 mg/kg & $105,47 \pm 1,89$ & $159,39 \pm 1,24$ & $154,9 \pm 0,72$ & $147,56 \pm 1,33$ & $139,84 \pm 1,51$ & $126,66 \pm 1,19$ & 113,6 \\
\hline Extracto $400 \mathrm{mg} / \mathrm{kg}$ & $103,53 \pm 2,23$ & $159,89 \pm 1,14$ & $147,56 \pm 1,33$ & $139,84 \pm 1,51$ & $126,66 \pm 1,19$ & $126,66 \pm 1,19$ & $109,43 \pm 1,58$ \\
\hline Extracto 600 mg/kg & $107,06 \pm 1,64$ & $162,74 \pm 1,87$ & $137,77 \pm 1,28^{c}$ & $124,34 \pm 1,12^{c}$ & $113,63 \pm$ & $106,51 \pm 1,92^{c}$ & $109,43 \pm 1,58$ \\
\hline Enalapril $75 \mathrm{mg} / \mathrm{kg}$ & $107,78 \pm 1,36$ & $153,19 \pm 1,12$ & $144,57 \pm 0,71$ & $133,62 \pm 0,58$ & 124,34 & $113,63 \pm 0,42$ & $106,51 \pm 1,92$ \\
\hline \multicolumn{8}{|c|}{$\begin{array}{l}\text { Los valores son expresados como media } \pm \text { error estándar. PB=Presión Basal, PPI=Presión Postinducción, PS=Presión Sistólica según día de medición }(1,5,10,15,20) \text {. } \\
\text { Existe diferencia significativa entre las medias de los grupos con respecto al grupo control sin L-NAME }(p<0,05) \text { (a), con respecto al grupo control con L-NAME } \\
(p<0,05) \text { (b) y existe diferencia significativa entre las medias del grupo de } 600 \mathrm{mg} / \mathrm{kg} \text { con respecto a los grupos de } 200 \mathrm{mg} / \mathrm{kg}, 400 \mathrm{mg} / \mathrm{kg} \text { y enalapril }(p<0,05) \text { (c). }\end{array}$} \\
\hline Tratamiento & PB & PPI & PD1 & PD5 & PD10 & PD15 & PD20 \\
\hline Control sin L-NAME & $85,11 \pm 1,81$ & $83,9 \pm 1,67^{a}$ & $79,57 \pm 1,70$ & $78,81 \pm 1,58$ & $79,45 \pm 2,27$ & $81,99 \pm 1,09$ & $81,6 \pm 1,32$ \\
\hline Control con L-NAME & $84,14 \pm 2,3$ & $134,74 \pm 2,72$ & $136,16 \pm 4,10^{b}$ & $125,4 \pm 1,94^{b}$ & $127,09 \pm 2,55^{b}$ & $119,17 \pm 1,53^{b}$ & $103 \pm 1,08^{b}$ \\
\hline Extracto 200 mg/kg & $81,59 \pm 2,01$ & $133,63 \pm 2,48$ & $125,4 \pm 1,94$ & $127,09 \pm 2,55$ & $119,17 \pm 1,53$ & $103 \pm 1,08$ & $87,14 \pm 3,24$ \\
\hline Extracto $400 \mathrm{mg} / \mathrm{kg}$ & $79,53 \pm 1,96$ & $138,34 \pm 2,09$ & $127,09 \pm 2,55$ & $119,17 \pm 1,53$ & $103 \pm 1,08$ & $103 \pm 1,08$ & $82,6 \pm 2,2$ \\
\hline Extracto $600 \mathrm{mg} / \mathrm{kg}$ & $83,46 \pm 2,45$ & $135,25 \pm 1,39$ & $96,94 \pm 1,69^{c}$ & $85,64 \pm 2,06^{c}$ & $87,14 \pm 3,24^{c}$ & $79,62 \pm 2,78^{c}$ & $82,6 \pm 2,2$ \\
\hline Enalapril $75 \mathrm{mg} / \mathrm{kg}$ & $80,76 \pm 1,84$ & $129,28 \pm 4,71$ & $119,87 \pm 1,90$ & $95,06 \pm 1,99$ & $85,64 \pm 2,06$ & $87,14 \pm 3,24$ & $79,62 \pm 2,78$ \\
\hline
\end{tabular}

Los valores son expresados como media \pm error estándar. PB=Presión Basal, $\mathrm{PPI}=$ Presión Postinducción, $\mathrm{PD}=$ Presión Diastólica según día de tratamiento $(1,5,10,15,20)$. Existe diferencia significativa entre las medias de los grupos con respecto al grupo control sin L-NAME $(p<0,05)(a)$, con respecto al grupo control con L-NAME $(p<0,05)$ (b) y existe diferencia significativa entre las medias del grupo de $600 \mathrm{mg} / \mathrm{kg}$ con respecto a los grupos de $200,400 \mathrm{mg} / \mathrm{kg}$ y enalapril $(p<0,05)(\mathrm{c})$.

Tabla 4. Efecto antihipertensivo sobre la presión arterial media del extracto metanólico a ratones con hipertensión inducida por L-NAME.

\begin{tabular}{|c|c|c|c|c|c|c|c|}
\hline Tratamiento & PB & PPI & PM1 & PM5 & PM10 & PM15 & PM20 \\
\hline Control sin L-NAME & $92,33 \pm$ & $5 \pm$ & $88,1 \pm 1,51$ & $87,94 \pm 1,33$ & $89,32 \pm 1,67$ & $91,9 \pm 0,92$ & $90,94 \pm 1,05$ \\
\hline Cont & $91,76 \pm$ & $143,62 \pm 1,92$ & 143,79 & $135,68 \pm 1,27$ & $134,69 \pm 3,74$ & $126,16 \pm 1,28$ & 110,7 \\
\hline Extracto & $89,6 \pm 1,86$ & $2,2 \pm 1,48$ & 1,27 & $134,69 \pm 3,74$ & $126,16 \pm 1,28$ & 110,7 & $93,7 \pm$ \\
\hline Extr: & 87,55 & 30 & 134,6 & 126,1 & 110,7 & 110,7 & 91 \\
\hline Extr & $91 \pm 2,22$ & $141,82 \pm 1,68$ & 110,1 & 98,5 & 93,7 & 88,87 & 91, \\
\hline Enalapril $75 \mathrm{mg} / \mathrm{kg}$ & $89,64 \pm 1,54$ & $139,32 \pm 4,13$ & $125,51 \pm 1,74$ & $107,62 \pm 1,27$ & $98,57 \pm 1,49$ & $93,7 \pm 1,03$ & $88,87 \pm 2,23$ \\
\hline
\end{tabular}

Los valores son expresados como media \pm error estándar. PB=Presión Basal, PPI=Presión Postinducción, PM=Presión Media según día de tratamiento (1, 5, 10, 15, 20). Existe diferencia significativa entre las medias de los grupos con respecto al grupo control sin L-NAME $(p<0,05)(a)$, con respecto al grupo control con L-NAME $(p<0,05)$ (b) y existe diferencia significativa entre las medias del grupo de $600 \mathrm{mg} / \mathrm{kg}$ con respecto a los grupos de 200, $400 \mathrm{mg} / \mathrm{kg}$ y enalapril $(p<0,05)(\mathrm{c})$.

cada caso, se hizo diariamente, vía oral, por 6o días, luego de lo cual, se extrajo sangre por punción cardiaca para realizar análisis a nivel hematológico (recuento de leucocitos) y bioquímico (glucosa, urea, transaminasa, fosfatasa alcalina, colesterol total, HDL-colesterol y triglicéridos). Luego se sacrificaron los animales con pentobarbital a dosis de $100 \mathrm{mg} / \mathrm{kg}$, según normas internacionales del cuidado de animales de experimentación ${ }^{11-12}$, para determinar la presencia de cambios histológicos en relación al grupo control a través del examen anatomopatológico de riñón, hígado, corazón, cerebro y pulmón ${ }^{13}$.

\section{Análisis estadístico}

Los resultados obtenidos fueron analizados mediante el test de Student (media y error estándar) y se aplicó el test de Tukey para las comparaciones entre los grupos, considerándose estadísticamente significativo para un $p<0,05$.

\section{RESULTADOS}

\section{Del análisis fitoquímico Ver tabla 1.}

De la determinación del efecto antihipertensivo

Ver tablas 2-4. normotensas por 60 días.

\section{Determinación de la toxicidad crónica}

Ver tablas 5 y 6.

\section{DISCUSIÓN}

En el análisis fitoquímico (tabla 1) el extracto metanólico mostró la presencia de compuestos fenólicos, flavonoides, taninos, alcaloides y quinonas. Estos resultados concuerdan con lo obtenido por Lastra y Yañez 14, quienes determinaron la presencia de flavonoides,

Tabla 5. Niveles de los datos bioquímicos al administrar el extracto metanólico a ratas

\begin{tabular}{lcccc}
\hline \multicolumn{1}{c}{ Tratamiento } & Glucosa & Colesterol total & HDL-Colesterol & Triglicéridos \\
\hline Control & $87,5 \pm 2,2$ & $158,75 \pm 10,2$ & $39 \pm 1,3$ & $147,25 \pm 11,7$ \\
Extracto $400 \mathrm{mg} / \mathrm{kg}$ & $91,62 \pm 3,8^{\text {a }}$ & $179,75 \pm 6,9^{\mathrm{b}}$ & $37,75 \pm 2,7^{\mathrm{b}}$ & $145,62 \pm 7,6^{\mathrm{a}}$ \\
Extracto $600 \mathrm{mg} / \mathrm{kg}$ & $89,75 \pm 3,3^{\mathrm{a}}$ & $161,37 \pm 7,8$ & $46 \pm 2,8$ & $131,75 \pm 13,6^{\mathrm{a}}$ \\
\hline
\end{tabular}

Los valores son expresados como media \pm error estándar. (a) No Existe diferencia significativa entre las medias de los grupos con respecto al grupo control $(p<0,05)$. (b) Existe diferencia significativa entre la media con respecto al grupo control y grupo de $600 \mathrm{mg} / \mathrm{kg}(p<0,05)$.

Tabla 6. Niveles de los datos bioquímicos y hematológicos al administrar el extracto metanólico a ratas normotensas por 60 días.

\begin{tabular}{lcccc}
\hline \multicolumn{1}{c}{ Tratamiento } & Urea & TGP & $\begin{array}{c}\text { Fosfatasa } \\
\text { alcalina }\end{array}$ & Leucocitos \\
\hline Control & $19,5 \pm 2,8^{\text {a }}$ & $17,25 \pm 3,7^{\text {a }}$ & $143,75 \pm 10,7^{\text {a }}$ & $7872,5 \pm 840^{\text {a }}$ \\
Extracto $400 \mathrm{mg} / \mathrm{kg}$ & $20,87 \pm 2,1$ & $22,5 \pm 5,3$ & $139 \pm 4,2$ & $6776,25 \pm 524$ \\
Extracto $600 \mathrm{mg} / \mathrm{kg}$ & $16,75 \pm 1,2$ & $24,12 \pm 5,2$ & $119,5 \pm 8,8$ & $7103,75 \pm 778,1$ \\
\hline
\end{tabular}

Los valores son expresados como media \pm error estándar. (a) No Existe diferencia significativa entre las medias de los grupos con respecto al grupo control $(p<0,05)$. 
compuestos fenólicos, saponinas y taninos en el extracto hidroalcohólico seco de la misma planta. Asimismo, en el extracto acuoso de otra especie del mismo género, Calceolaria tripartita, Tomas et al ${ }^{\mathbf{1 5}}$, encontraron la presencia de diterpenos, cumarinas y alcaloides, lo que evidencia concordancias con los hallazgos aquí reportados. Se debe señalar que la especie vegetal del presente estudio pertenece a la familia Scrofulariaceae, donde también se encuentran Digitalis lanata y Digitalis purpurea,que en su composición fitoquímica presentan heterósidos cardiotónicos 16.

Está demostrado que la administración durante siete días consecutivos de L-NAME, a dosis de $130 \mathrm{mg} /$ kg por vía oral, produce aumento de la presión arterial 10. L-NAME inhibe irreversiblemente la enzima óxido nítrico sintetasa (NOS) ocasionando disminución de la producción de óxido nítrico en el endotelio a partir de L-arginina, vasoconstricción, aumento de la liberación de renina e hipertensión arterial ${ }^{17}$. Según los resultados de la presente investigación, el extracto metanólico de Calceolaria myriophylla en dosis de 200, 400 y 600 $\mathrm{mg} / \mathrm{kg}$ produjo actividad antihipertensiva a nivel de presión arterial sistólica, diastólica y media a lo largo del tratamiento durante 30 días, como se indica en las tablas 2, 3 y 4 . Esta actividad se hizo sostenible a partir del décimo día de tratamiento. Hubo diferencia significativa $(p<0,05)$ entre los grupos experimentales en relación al grupo control con L-NAME. Un dato importante a resaltar es que el grupo experimental a dosis de $600 \mathrm{mg} /$ $\mathrm{kg}$ presentó mejor efecto antihipertensivo, sin diferencias significativas con los demás, superando incluso al grupo con enalapril $(p<0,05)$.

Estudios como el presente, en plantas medicinales con actividad antihipertensiva, desarrollados en modelos preclínicos y clínicos, muestran constituyentes químicos responsables de dicha actividad como son los flavonoides, compuestos fenólicos y saponinas esteroidales $\mathbf{1 6}$. Los flavonoides y compuestos fenólicos actúan como vasodilatadores ${ }^{18}$ e inhibidores del sistema reninaangiotensina ${ }^{19}$, lo cual explicaría la acción diurética atribuida por la medicina tradicional ${ }^{16,20}$.

En la evaluación de la toxicidad crónica por dosis repetidas de 400 y $600 \mathrm{mg} / \mathrm{kg}$ vía oral, por 60 días (tablas 5 y 6), se determinó que el extracto metanólico de Calceolaria myriophylla no presentó cambios bioquímicos ni hematológicos estadísticamente significativos $(p>0,05)$ tomando como referencia al grupo control, a excepción de los niveles de HDL-colesterol, que aumentaron, y de colesterol sérico, que disminuyeron (con significatividad estadística el primero y si ella el segundo), a la dosis de $600 \mathrm{mg} / \mathrm{kg}$.

En la evaluación anatomopatológica del riñón, corazón, cerebro, hígado y pulmón no se presentaron cambios histopatológicos anormales con relación al grupo control. Esto indicaría que existe un margen de seguridad a nivel de toxicidad, sin embargo, se necesita confirmar con estudios más prolongados, porque al ser la hipertensión arterial una enfermedad crónica, implica la administración de medicamentos -fitoterapéuticos o nopor tiempo indefinido.

\section{CONCLUSIONES}

El extracto metanólico de Calceolaria myriophylla (zapatilla) contiene compuestos fenólicos y flavonoides; asimismo produjo actividad antihipertensiva sobre la presión arterial sistólica, diastólica y media en ratones con hipertensión inducida por L-NAME, sin efecto tóxico a nivel hematológico, bioquímico y anatomopatológico.

\section{REFERENCIAS BIBLIOGRÁFICAS}

1. Vega T, Lozano J, Álamo R, Lleras S. Prevalencia de la hipertensión arterial en la población de Castilla y León. Gac Sanit. 2008; 22(4): 330-6.

2. Rivas J, Gutiérrez C, Rivas J. Tratamiento y costos farmacológicos de la hipertensión arterial no complicada. Rev Soc Peru Med Interna. 2007; 20(4): 139-44.

3. Mantilla J, Olazábal O. Las plantas medicinales de nuestra Madre Tierra. Instituto de Ecología y Plantas Medicinales - IEPLAM, Perú. Cusco; 2001.

4. Roesrch C, Van der Hoogte L. Plantas medicinales en el sur andino del Perú. Tomo 2. Cusco: Koeltz Scientific Books; 1994.

5. Ríos C. Evaluación de las plantas de interés etnomedicinal en la comunidad de Choccopía del distrito de ColquepataPaucartambo. [Seminario Curricular]. Facultad de Biología. Universidad Nacional San Antonio Abad del Cusco, Perú. Cusco; 2002.

6. Sharapin N. Fundamento de tecnología de productos fitoterapéuticos. Santa Fe de Bogotá: Publicación del programa Andrés Bello - Programa Iberoamericano de Ciencia y Tecnología para el Desarrollo, 2000.

7. Lock de Ugaz O. Investigación fitoquímica - Métodos en el estudio de productos naturales. Lima: Fondo Editorial de la Pontificia Universidad Católica del Perú; 1988.

8. Arroyo J, Rojas J, Chenguayen J. Manual de Modelos Experimentales de Farmacología. Lima: Publicaciones ASDIMOR, 2004.

9. Sharifi A, Akbarloo N, Darabi R. Investigation of local ACE activity and structural alterations during development of L-NAME induced hypertension. Pharmacological Research. 2005; 52(5): 438-44.

10. Obst M, Gross V, Janke J, Wellner M, Schneider W, Luft F. Pressure natriuresis in AT2 receptor-deficient mice with L-NAME hypertension. J Am Soc Nephrol, 2003; 14(2): 303-10.

11. Close B, Banister K, Baumans V, Bernoth E, Bromage N, Bunyan J, et al. Recomendaciones para la Eutanasia de los Animales de Experimentación: Parte 1. Oxford: Comisión Europea, 1986.

12. Ramírez J, Palacios M, Tamayo O, Jaramillo R, Gutierrez O. Acute and subacute toxicity of Salvia scutellarioides in mice and rats. J Ethnopharmacol. 2007; 109(2): 348-53.

13. Qin Y, Wu X, Huang W, Gong G, Li D, He Y, Zhao Y. Acute toxicity and sub-chronic toxicity of steroidal saponins from Dioscorea zingiberensis C.H.Wright in rodents. J Ethnopharmacol, 2009; 126(3): 543-50.

14. Lastra Y, Yáñez B. Efecto Gastroprotector de Gamochaeta americana (Mill) Wedell (K'eto K'eto) y Calceolaria myriophylla Kraenz (Ayaq Zapatilla) sobre lesiones 
gástricas inducidas por indometacina en ratas. [Tesis para optar al Título Profesional de Químico Farmacéutico]. Facultad de Ciencias Químicas, Físicas, Matemáticas, Farmacia e Informática. Universidad Nacional de San Antonio Abad del Cusco. Cusco, 2004.

15. Tomas G, Arroyo J, Rojas J, Chenguayen J, Aguirre R, Huamán J, et al. Actividad farmacológica de los extractos acuoso y etanólico de Calceolaria tripartita (sachaberros). Revista Peruana de Química e Ingeniería Química, 2006; 9(1): 10-3.

16. Kuklinski C. Farmacognosia. Estudio de las drogas y sustancias medicamentosas de origen natural. Barcelona: Editorial Omega S.A, 2003.

17. Maicas C, Lázaro E, Alcalá J, Hernández P, Rodríguez L. Etiología y fisiopatología de la hipertensión arterial esencial. Monocardio. 2003; 3(V): 141-6o.

18. Rojas J, Ronceros S, Palomino R, Tomás G, Chenguayen J. Efecto antihipertensivo y dosis letal 50 del jugo del fruto y del extracto etanólico de las hojas de Passiflora edulis (maracuyá), en ratas. An Fac Med Lima. 2006; 67(3): 206-13.

19. Fatehi M, Saleh T, Fatehi-Hassanabad Z, Farrokhfal K, Jafarzadeh M, Davodi S. A pharmacological study on Berberis vulgaris fruit extract. J Ethnopharmacol. 2005; 102(1): 46-52.

2o. Maghrani M, Zeggwagh A, Haloui M, Eddouks M. Acute diuretic effect of aqueous extract of Retama raetam in normal rats. J Ethnopharmacol. 2005; 99(1):31-5.

Manuscrito recibido el: 14/03/2016

Aceptado para su publicación el: 19/o9/2016

\section{Correspondencia}

Nombre: Martin Condorhuamán Figueroa

Dirección: Jr. Puno 1002, Lima 1.

e-mail: marcofi71@yahoo.es 